\title{
Predicted Oxidation of CO Catalyzed by Au Nanoclusters on a Thin Defect-Free MgO Film Supported on a Mo(100) Surface
}

\author{
Chun Zhang, Bokwon Yoon, and Uzi Landman* \\ School of Physics, Georgia Institute of Technology, Atlanta, Georgia 30332-0430
}

Received December 11, 2006; E-mail: uzi.landman@physics.gatech.edu

Recently there has been a surge in research pertaining to the physical and chemical properties of gold nanoclusters. Unlike supported particles of larger sizes, or extended solid surfaces ${ }^{1,2}$ small metal clusters adsorbed on support materials were found to exhibit unique properties that originate from the highly reduced dimensions of the individual metal aggregates. ${ }^{3-8}$ In particular we note here joint experimental studies and theoretical investigations ${ }^{5-8}$ using first-principles simulations on size-selected small gold clusters, $\mathrm{Au}_{n}$ ( $20 \geq n \geq 2$ ), adsorbed on a well characterized metal-oxide support under ultrahigh-vacuum conditions. These studies revealed that cluster morphology, dimensionality, dynamical structural fluxionality, electronic structure, and charge state, as well as the state of the metal-oxide surface (specifically, defect-rich $\mathrm{MgO}(100)$ surfaces containing oxygen-vacancy F centers, or defect-poor surfaces), govern the catalytic activity. Particular emphasis has been put $^{5-8}$ on the low-temperature (as low as $140 \mathrm{~K}$ ) oxidation of CO, catalyzed by $\mathrm{Au}_{n}$ clusters with $7<n<25$ atoms that are adsorbed on F-center-rich magnesia surfaces. In these investigations charging of the adsorbed metal cluster through partial electron transfer from the substrate F-center defect and subsequent occupation of the antibonding $2 \pi^{*}$ orbital of $\mathrm{O}_{2}$ leading to activation of the $\mathrm{O}-\mathrm{O}$ bond of the molecule adsorbed on the cluster (resulting in formation of peroxo or superoxo states), have been identified as underlying the catalytic activity.

Here we show results of first-principles investigations aiming at tuning and controlling the catalytic activity of gold nanoclusters through the design of the underlying support. We show that gold clusters adsorbed on a very thin (2 layers) defect-free $\mathrm{MgO}$ film, which is itself supported on $\mathrm{Mo}(100),{ }^{9}$ may serve as model catalysts for the low-barrier oxidation of $\mathrm{CO}$. The origin of the emergent activity of the nanoclusters is a dimensionality crossover of the adsorbed gold clusters, from inactive three-dimensional (3D) optimal structures on thick $\mathrm{MgO}$ films to catalytically active 2D ones for sufficiently thin $\mathrm{MgO}$ films (less than $1 \mathrm{~nm}$ in thickness) supported on $\mathrm{Mo}(100)$. The increased gold wetting propensity on the $\mathrm{MgO} / \mathrm{Mo}(100)$ surface originates from electrostatic interaction between the underlying metal and metal-induced excess electronic charge accumulated at the cluster interface with the metal-oxide film. ${ }^{10}$ The excess interfacial charge is predicted here to activate $\mathrm{O}_{2}$ molecules adsorbed at the interfacial periphery of the $2 \mathrm{D}$ gold island with the $\mathrm{MgO} / \mathrm{Mo}(100)$ surface. This activation, which weakens greatly the $\mathrm{O}-\mathrm{O}$ bond, lowers rather remarkably the barrier for reaction of the activated molecule with $\mathrm{CO}$ and the subsequent emission of $\mathrm{CO}_{2}$.

Our first-principles calculations are based on a density functional theory approach ${ }^{11,12}$ with exchange and correlation energy corrections included through a generalized gradient approximation (GGA). ${ }^{13}$ Such calculations have been shown to give very accurate bond lengths (up to $1 \%$ too long), and reaction energy barriers that are accurate to within $30 \%$ (usually too low) (see page 87 of ref 8). A plane wave basis is used with a cutoff kinetic energy of 30

2228 - J. AM. CHEM. SOC. 2007, 129, 2228-2229
Ry, and ultrasoft pseudopotentials (scalar relativistic for $\mathrm{Au})^{14}$ are employed with $\Gamma$-point sampling of the Brillouin zone. In structural relaxations corresponding to minimization of the total energy, convergence is achieved when the forces on the atoms are less than $0.001 \mathrm{eV} / \AA$.

In modeling the metal-supported $\mathrm{MgO}$ films we use a four-layer Mo(100) slab (lattice constant of $3.15 \AA$ ) of thickness $4.64 \AA$, which has been found to reproduce (in its middle) the bulk electronic properties of Mo. ${ }^{15}$ We adsorb the planar $\mathrm{Au}_{20}$ clusters on a $\mathrm{MgO}$ / $\mathrm{Mo}(100)$ with $5 \times 6$ unit cells of the Mo(100) surface; for the 3Dtetrahedral cluster the dimensions of the $\mathrm{MgO}$ surface (without the metal support) are the same, and the bottom layer of the metal oxide is held fixed. In all calculations, the periodically replicated slabs are separated [in the (100) direction] by a vacuum region of $20 \AA$. In structural optimizations all the atoms of the adsorbed gold clusters, the $\mathrm{MgO}$ thin film, and the first two layers of the Mo substrate are allowed to relax. In calculations involving the tetrahedral gold structure, the $\mathrm{MgO}(100)$ crystalline surface was modeled by a two-layer $\mathrm{MgO}(100)$ slab which is sufficiently thick both to reproduce the properties of the bare $\mathrm{MgO}$ surface $^{16}$ and to obtain converged results (with respect to the number of $\mathrm{MgO}$ layers) for the energetics of adsorbed Au clusters ${ }^{17}$ (for further details see ref 10).

The gas-phase optimal 3D tetrahedral $\mathrm{Au}_{20}$ cluster ${ }^{18}$ maintains its structure on the $\mathrm{MgO}(100)$ surface, with a $1.2 \mathrm{eV}$ advantage over the planar structure. However, this cluster is found to adsorb $\mathrm{O}_{2}$ only weakly $(0.34 \mathrm{eV}$ on the top apex atom of the pyramid with $(d(\mathrm{O}-\mathrm{O})=1.28 \AA$ remaining close to the gas-phase value), and no binding is found at peripheral sites of the gold $/ \mathrm{MgO}$ interface; when $\mathrm{CO}$ is preadsorbed to the top apex $\mathrm{Au}$ atom $(0.7$ eV) no coadsorption of $\mathrm{O}_{2}$ occurs.

In light of the above inactivity of the 3D structure we focused our investigations on $\mathrm{Au}_{20}$ adsorbed on a two-layer $\mathrm{MgO} / \mathrm{Mo}(100)$. Here the planar isomer is more stable than the $3 \mathrm{D}$ one by $3.3 \mathrm{eV}$, with the enhanced stability resulting from penetration of metal states through the thin metal oxide film and charge accumulation at the cluster/ $\mathrm{MgO}$ interface (up to $1 \mathrm{e}$ for $\mathrm{Au}_{20}$ ). ${ }^{10}$ Furthermore, in this configuration the $\mathrm{O}_{2}$ adsorbs relatively strongly $(1.35 \mathrm{eV})$ at the cluster periphery (Figure 1), and the process is accompanied by transfer of electronic charge ${ }^{19}$ (about 1.3 e) into the antibonding $2 \pi^{*}$ orbital leading to activation of the $\mathrm{O}-\mathrm{O}$ bond of the adsorbed molecule into a peroxo state with $d(\mathrm{O}-\mathrm{O})=1.52 \AA$ and no spin polarization (that is a spin-singlet state).

We explored two mechanism for the reaction of $\mathrm{CO}$ with the activated oxygen molecule. One involves a coadsorbed $\mathrm{CO}$ molecule (Langmuir-Hinshelwood, LH), and in the other one the reactant $\mathrm{CO}$ molecules approach the activated $\mathrm{O}_{2}$ directly from the gas phase (Eley-Rideal, ER). Various CO coadsorption sites were explored. First we examined adsorption to the gold cluster, yielding relaxed adsorption configurations with $\mathrm{CO}$ binding energies ranging from $0.8 \mathrm{eV}$ (CO binding to a peripheral $\mathrm{Au}$ atom that is nearest-neighbor 


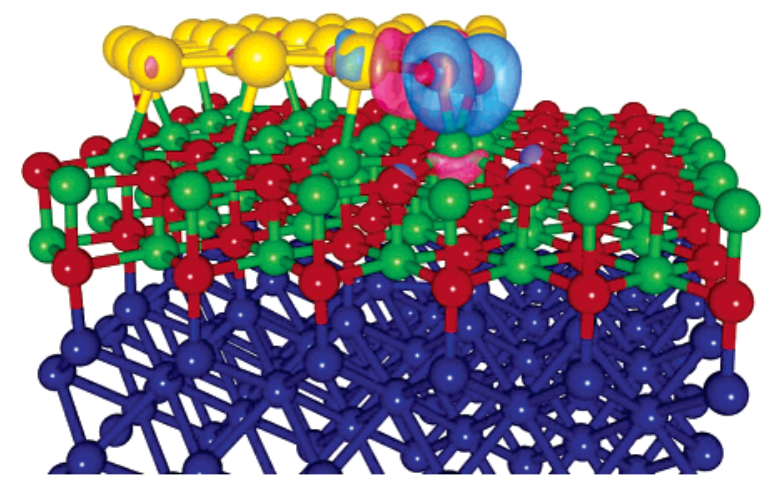

Figure 1. Two-dimensional $\mathrm{Au}_{20}$ island (yellow on line) adsorbs on a twolayer $\mathrm{MgO}$ film ( $\mathrm{O}$ atoms in red and $\mathrm{Mg}$ in green) supported on $\mathrm{Mo}(100)$ (blue on line), with a coadsorbed $\mathrm{O}_{2}$ molecule. Superimposed we show an isosurface of the excess electronic charge (light blue on line) illustrating activation of the adsorbed molecule through population of the antibonding $2 \pi^{*}$ orbital.

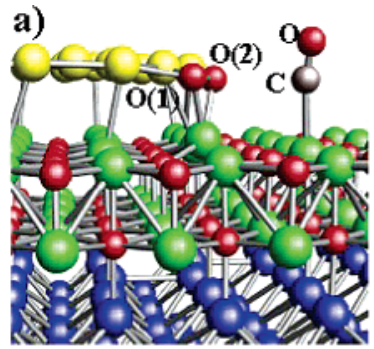

c)

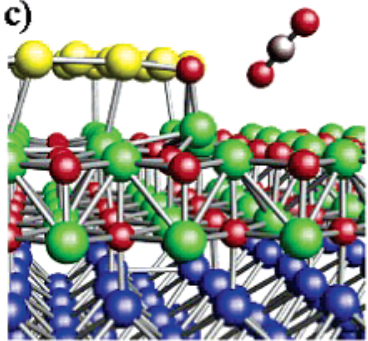

b)

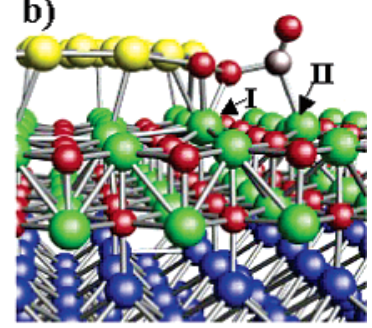

d)

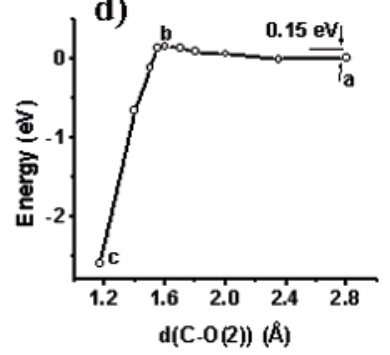

Figure 2. $(\mathrm{a}-\mathrm{c})$ Configurations of the two-dimensional $\mathrm{Au}_{20}$ island shown in Figure 1 (the color scheme is the same as in Figure 1) with coadsorbed $\mathrm{O}_{2}$ (the atoms marked $\mathrm{O}(1)$ and $\left.\mathrm{O}(2)\right)$ and $\mathrm{CO}(\mathrm{C}$ atom in gray online): (a) the initial optimized configuration; $d(\mathrm{O}(1)-\mathrm{O}(2))=1.52 \AA, d(\mathrm{C}-\mathrm{O}(2))=$ $2.85 \AA$; (b) the transition state (the nearest-neighbor $\mathrm{Mg}$ atoms are marked as I and II); distances are $d(\mathrm{O}(1)-\mathrm{O}(2))=1.55 \AA, d(\mathrm{C}-\mathrm{O}(2))=1.60 \AA$, $d(\mathrm{C}-\mathrm{O})=1.18 \AA, d(\mathrm{C}-\mathrm{Mg}(\mathrm{II}))=2.30 \AA$; $(\mathrm{c})$ a configuration illustrating formation and desorption of $\mathrm{CO}_{2}$; (d) the total energy profile along the $\mathrm{C}-\mathrm{O}(2)$ reaction coordinate, with the zero of the energy scale taken for configuration a. The sharp drop past the barrier top corresponds to $\mathrm{CO}_{2}$ formation.

to the $\mathrm{Au}$ atom bonded to the $\mathrm{O}_{2}$ ) down to a vanishingly small binding for a nonperiphery nearest-neighbor $\mathrm{Au}$ atom on the flat gold island. However, the calculation of the barrier ${ }^{20}$ for reaction between the reactants (with $\mathrm{CO}$ at the peripheral site) yielded a high value of close to $1.0 \mathrm{eV}$.

We have tested also several $\mathrm{CO}$ coadsorption sites located on the $\mathrm{MgO}$ surface in the vicinity of the adsorbed $\mathrm{O}_{2}$. The adsorption geometry shown in Figure $2 \mathrm{a}$ is characterized by a $\mathrm{CO}$ binding energy of $0.4 \mathrm{eV}$. Starting from this coadsorption configuration, we calculate the $\mathrm{LH}$ reaction barrier leading to formation of $\mathrm{CO}_{2}$ to be $0.15 \mathrm{eV}$; the transition state is shown in Figure $2 \mathrm{~b}, \mathrm{CO}_{2}$ formation in Figure 2c, and the energy profile along the reaction coordinate is displayed in Figure 2d. The product molecule desorbs readily in the course of the reaction (the binding energy of $\mathrm{CO}_{2}$ to a $\mathrm{Mg}$ site on the $\mathrm{MgO}(100)$ surface is merely $0.14 \mathrm{eV}$ ). Alternatively, we considered several reaction trajectories that follow the ER mechanism. Some of these trajectories yielded formation of $\mathrm{CO}_{2}$ with small $(0.2 \mathrm{eV}$, see Supporting Information), or vanishingly small, barriers, depending on the angle of approach.

In summary, with the use of first-principles simulations we predict low-barrier $\mathrm{CO}$ oxidation reactions to occur via a Langmuir-Hinshelwood or an Eley-Rideal mechanism on 2D gold nanocluster islands adsorbed on very thin (two layers) $\mathrm{MgO}$ films that are supported on $\mathrm{Mo}(100)$. Underlying the catalytic activity, that is predicted to occur even in the absence of F-center defects, is the excess electronic charge at the gold cluster/magnesia interface. This results from penetration of metal states through the MO thin film. We expect these results to provide the impetus for future experiments as well as the further development of methods for controlling the interfacial charge (and consequently the chemical reactivity) in nanocatalytic systems, for example, through the use of applied fields.

Acknowledgment. This research was supported by the U.S. AFOSR and the DOE. Computations were performed at the DOE National Energy Research Scientific Computing Center at the Lawrence Berkeley National Laboratory (NERSC) and at the Georgia Tech Center for Computational Materials Science.

Supporting Information Available: Energy profile, transition state, and final configuration of one of the Eley-Rideal mechanisms. This material is available free of charge via the Internet at http://pubs.acs.org.

\section{References}

(1) Henry, C. R. Surf. Sci. Rep. 1998, 31, 231

(2) Ertl, G.; Freund, H.-J. Phys. Today 1999, 52 (1), 32

(3) Haruta, M. Catal. Today 1997, 36, 153.

(4) Valden, M.; Lai, X.; Goodman, D. W. Science 1998, 281, 1647.

(5) Sanchez, A.; Abbet, S.; Heiz, U.; Schneider, W.-D.; Häkkinen, H.; Barnett, R. N.; Landman, U. J. Phys. Chem. A 1999, 103, 9573.

(6) Häkkinen, H.; Abbet, S.; Sanchez, A.; Heiz, U.; Landman, U. Angew. Chem., Int. Ed. 2003, 42, 1297.

(7) Yoon, B.; Häkkinen, H.; Landman, U.; Wörz, A. S.; Antonietti, J.-M.; Abbet, S.; Judai, K.; Heiz, U. Science 2005, 307, 403.

(8) Heiz, U.; Landman, U. Nanocatalysis; Springer: New York, 2006.

(9) This scheme is commonly employed in experimental studies of $\mathrm{Au}$ nanstructures adsorbed on MgO films: see Wu, M.-C.; Corncillc, J. S.; Estrada, C. A.; He, J. W.; Goodman, D. W. Chem. Phys. Lett. 1991, 182, 472 (see also refs 5 and 8 ).

(10) Ricci, D.; Bongiorno, A.; Paccioni, G.; Landman, U. Phys. Rev. Lett. 2006, 97, 36106.

(11) Kresse, G.; Hafner, J. Phys. Rev. B 1993, 47, R558.

(12) Kresse, G.; Furthmuller, J. Phys. Rev. B 1996, 54, 11169.

(13) Perdew, J. P.; Chevary, J. A.; Vosko, S. H.; Jackson, K. A.; Pederson, M. R.; Singh, D. J.; Fiolhais, C. Phys. Rev. B 1992, 46, 6671.

(14) Vanderbilt, D. Phys. Rev. B 1990, 41, 7892.

(15) Giordano, L.; Baistrocchi, M.; Pacchioni, G. Phys. Rev. B 2005, 72, 115403.

(16) Giordano, L.; Goniakowski, J.; Pacchioni, G. Phys. Rev. B 2003, 67, 045410.

(17) Molina, L. M; Hammer, B. Phys. Rev. B 2004, 69, 155424.

(18) Li, J.; Li, X.; Zhai, H.-J.; Wang, L.-S. Science 2003, 299, 864

(19) An estimate of the excess charge on the adsorbed oxygens is obtained by integrating the charge density in the two oxygen Voronoi cells and subtracting the number of valence electrons (6) of a free oxygen. The Voronoi cell is a generalization of the Wigner-Seitz cell, see the definition in Okabe, A.; Boots, B.; Sugihara, K. Spatial Tesellations: Concepts and Applications of Voronoi Diagrams, 2nd ed; Wiley: New York, 2000.

(20) Reaction barriers were determined via constrained total energy minimization with all degrees of freedom allowed to relax along a path where the varied reaction coordinate is the distance between the $\mathrm{C}$ atoms of the $\mathrm{CO}$ and the nearest oxygen atom of the activated molecule.

JA0684545 